\title{
Cohesive, frictional powders: contact models for tension
}

\author{
Stefan Luding
}

Received: 22 December 2006 / Published online: 27 March 2008

(C) The Author(s) 2008

\begin{abstract}
The contacts between cohesive, frictional particles with sizes in the range $0.1-10 \mu \mathrm{m}$ are the subject of this study. Discrete element model (DEM) simulations rely on realistic contact force models-however, too much details make both implementation and interpretation prohibitively difficult. A rather simple, objective contact model is presented, involving the physical properties of elastic-plastic repulsion, dissipation, adhesion, friction as well as rollingand torsion-resistance. This contact model allows to model bulk properties like friction, cohesion and yield-surfaces. Very loose packings and even fractal agglomerates have been reported in earlier work. The same model also allows for pressure-sintering and tensile strength tests as presented in this study.
\end{abstract}

Keywords Granular materials · Molecular dynamics (MD) and discrete elementmodel (DEM) force-laws · Friction . Rolling- and torsion-resistance $\cdot$ Adhesion .

Plastic deformation

\section{Introduction}

Cohesive, frictional, fine powders show a peculiar flow behavior that can be quantified by macroscopic bulk properties as, among others, cohesion, friction, yield and tensile strengths, dilatancy, stiffness, and anisotropy. The

S. Luding ( $\varangle)$

Multi Scale Mechanics, TS, CTW, UTwente,

P.O. Box 217, 7500 AE Enschede, Netherlands

e-mail: s.luding@utwente.nl

S. Luding

Particle Technology, Nanostructured Materials, DelftChemTech,

TNW, TUDelft, Julianalaan 136, 2628 BL Delft, Netherlands information propagation in such granular media is not completely understood, neither on the micro- nor on the macrolevel, especially when friction and other contact mechanisms are involved. Nevertheless, the macroscopic properties are controlled by the "microscopic" contact forces and torques, involving, e.g., contact adhesion or friction. Molecular dynamics (MD) or discrete element models (DEM) require the contact forces and torques as the basic input, to solve the equations of motion for all particles in the system. Alternative methods like event-driven MD $[38,39,41]$ or contact dynamics $[28,55,56,63,65]$ are based on further simplifications, like the assumption of instantaneous contacts or the perfect rigidity of particles, but will not be discussed here.

Research challenges involve not only the realistic quantitative and predictive simulation of many-particle systems, their experimental validation, but also the transition from the microscopic contact properties to the macroscopic flow behavior. This so-called micro-macro transition should allow to understand the collective flow behavior of many particles as function of their contact properties.

The goal of this paper is to provide a minimal set of contact models - as a compromise between a realistic and an easy to handle modeling approach. Naturally the contact model will be over-simplified, however, many details seem not to be important for the behavior on the macroscopic level. A single contact-model allows to simulate various systems and structures, as mentioned above. A better and deeper understanding of the relation between micro- and macro-properties will be facilitated by simpler contact models-fine-tuning can be achieved in a future step.

\subsection{Frictional contact models}

Typically the normal and tangential (frictional) contact forces are dealt with separately. While the former are subject of 
ongoing dispute, the latter are implemented in a commonly accepted way, based on the first realistic model for static friction, as introduced by Cundall and Strack $[6,12,39,64,73]$ : a virtual tangential spring is attached to each contact and evolves while the contact partners are moving and rotating, relative to each other, due to the contact force and the many other forces from other particles. Even though much more advanced models were discussed in the literature, related to the early works of Mindlin et al. [53,54], Derjaguin et al. [15], and Johnson et al. [26], the basic idea remains the same, being complemented by additional effects like, e.g., hysteresis, non-linearity, and others [27,72,83,84,93,94]. Advanced contact models are then applied to various situations in powder flow $[6,29,36,73,80,82,95,97]$. The present study will deal with the simplest linear visco-elastic tangential spring only, however, involving the possibility for different coefficients of static and dynamic friction as a new ingredient.

The tangential friction will lead to forces, but also to torques on the contact partners. Rolling- and torsion-resistance $[2,16,17,44,62,78]$ can play an important role in particle systems, since they also lead to torques, typically reducing the particles' freedom to rotate. This can be used to mimick the effects of surface roughness and non-spherical shapes to some extent [57-59], but naturally, non-spherical particles require more advanced algorithms $[31,52,89]$ —not discussed further in this study.

The present implementation of rolling- and torsionresistance is based on the same ideas as the model for static and dynamic friction - even the algorithm/subroutine for the evolution of the tangential spring can be used for rolling and torsion degrees of freedom-for both particle-particle and particle-wall contacts. Note that one has to assure that the contact models are objective, i.e., a rotation of the frame of reference must not affect the result.

\subsection{Normal contact modeling}

For fine particles, not only friction is relevant, but also adhesive contact properties due to van der Waals forces. Since effects like liquid and possibly permanent solid bridges are not subject of the present study, we refer to the detailed literature, see Refs. $[5,9,19,20,24,68,69,74,87,88,90]$ and references therein.

Also other phenomena are relevant for the normal force model: Due to the very small contact areas, already moderate forces will lead to plastic yield and plastic deformation of the material in the vicinity of the contact. This will lead to a larger contact area with increased stiffness and increased adhesion due to the van der Waals forces. Like in the case of friction, plenty of models are available, some of them based on visco-elasticity $[7,32,47,76]$ others on elastoplasticity $[25,27,83,84,93,94]$. For spheres, typically contact models in the spirit of Hertz $[4,22,35,60,66,70,79]$ seem appropriate—but only when the forces are small enough so that the yield stress is reached nowhere close to the contact area. For rather large metal spheres, the details of contact models are even measurable, when waves propagate along chains of particles $[10,11,49,75]$, and a Hertz based contact law is recommmended. However, Hertz models will not be discussed in this study, since finer powders only have a negligible range of elastic Hertz-like behavior [87] and, furthermore, are never perfectly spherical at the contact anyway. The present model is a piece-wise linear generalization of the hysteretic model ideas of Walton [94,96], involving plastic deformations, nonlinear stiffness and history dependent adhesion [43,44].

When contact overlaps/deformations become too large, the physics changes and the present model is limited by a simple linear force displacement branch with the maximal contact stiffness. This is convenient, since it allows to fix the time-step for numerical integration, however, the model becomes questionable in the regime of large deformations.

\subsection{Related issues in brief}

For techniques to perform the so-called micro-macro transition, see e.g. $[40,91,92]$ and references therein. The challenge here is to reduce the tremendous amount of information on the contact level, like contact-orientation and -force probability distribution functions [68], to the relevant macroscopic properties related to bulk-moduli, anisotropy and inhomogeneity in the contact network. The quest for a macroscopic constitutive model based on microscopic contact parameters is still ongoing.

Contact force measurements are rather simple for larger particles $[18,33,37]$, but for particles of micrometer size advanced techniques have to be applied, see e.g. $[8,20,30]$ and references therein. Even though contacts can have a temperature and time-dependent behavior as during sintering $[46,50,51]$, this will not be the issue of the present study. Since the model presented below allows for pressuresintering, a sample of particles can form a solid block, if compressed strong enough. The solid, sintered sample contains all memory of its history and the primary particles are still separate entities. Such a "granule" can then be examined by compressive and tensile tests - and all this without the much more complex modeling of non-spherical particles and without the often used beam-like models for contact adhesion and rolling resistance [89].

\section{Soft particle molecular dynamics (MD)}

Many-particle simulation methods like MD are also referred to as discrete element models (DEM) [3, 12,21,34,81,85,91]. 
They complement experiments on small "representative volume elements" (RVEs) by providing deep and detailed insight into the kinematics and dynamics of the samples examined. Large scale industrial applications, simulated particle by particle, are out of reach of DEM, since much more than the typical easy-to-deal-with million particles are involved in a silo or a dam.

\subsection{Discrete particle model}

The realistic and detailed modeling of the deformations of particles in contact with each other is much too complicated; therefore, we relate the interaction force to the overlap $\delta$ of two particles, see Fig. 1. In tangential direction, the forces and torques also depend on the tangential displacement and the relative rotations of the particle surfaces - different rotational degrees of freedom are responsible for sliding, rolling and torsion. Inter-particle forces based on the overlap and relative motion might not be sufficient to account for the inhomogeneous stress distribution inside the particles and possible multi-contact effects. Thus, the results presented here are of the same quality as the simplifying assumptions about the force-overlap relations made. However, it is the only way to model larger samples of particles with a minimal complexity of the contact properties, taking into account the relevant phenomena: non-linear contact elasticity, plastic deformation, and adhesion.

\subsection{Equations of motion}

Given the sum of forces $f_{i}$ acting on a particle $i$, either from other particles, or from walls, the problem is reduced to the integration of Newton's equations of motion for the translational and rotational degrees of freedom:

$m_{i} \frac{\mathrm{d}^{2}}{\mathrm{~d} t^{2}} \boldsymbol{r}_{i}=\boldsymbol{f}_{i}+m_{i} \boldsymbol{g}, \quad$ and $\quad I_{i} \frac{\mathrm{d}}{\mathrm{d} t} \boldsymbol{\omega}_{i}=\boldsymbol{q}_{i}$,

with the mass $m_{i}$ of particle $i$, its position $\boldsymbol{r}_{i}$ the total force $f_{i}=\sum_{c} f_{i}^{c}$, the acceleration due to volume forces like gravity $g$, the particles moment of inertia $I_{i}$, its angular velocity $\boldsymbol{\omega}_{i}$ and the total torque $\boldsymbol{q}_{i}=\boldsymbol{q}_{i}^{\text {friction }}+\boldsymbol{q}_{i}^{\text {rolling }}+\boldsymbol{q}_{i}^{\text {torsion }}$, as defined below.

The equations of motion are thus a system of $\mathcal{D}+\mathcal{D}$ $(\mathcal{D}-1) / 2$ coupled ordinary differential equations to be solved in $\mathcal{D}$ dimensions, with $\mathcal{D}=2$ or $\mathcal{D}=3$. With tools from numerical integration, as nicely described in textbooks as $[1,61,67]$, this is a straightforward exercise. The typically short-ranged interactions in granular media allow for optimization by using linked-cell (LC) or alternative methods in order to make the neighborhood search more efficient. In the case of interactions that range longer than contactinteractions, (e.g., charged particles or van der Waals type forces) this is not possible anymore, so that either a cut-off distance or more advanced methods for speed-up have to be applied.

\subsection{Normal contact force laws}

Two spherical particles $i$ and $j$, with radii $a_{i}$ and $a_{j}$, respectively, interact only if they are in contact so that their overlap

$\delta=\left(a_{i}+a_{j}\right)-\left(\boldsymbol{r}_{i}-\boldsymbol{r}_{j}\right) \cdot \boldsymbol{n}$

is positive, $\delta>0$, with the unit vector $\boldsymbol{n}=\boldsymbol{n}_{i j}=\left(\boldsymbol{r}_{i}-\right.$ $\left.\boldsymbol{r}_{j}\right) /\left|\boldsymbol{r}_{i}-\boldsymbol{r}_{j}\right|$ pointing from $j$ to $i$. The force on particle $i$, from particle $j$, at contact $c$, can be decomposed into a normal and a tangential part as $\boldsymbol{f}^{c}:=\boldsymbol{f}_{i}^{c}=f^{n} \boldsymbol{n}+f^{t} \boldsymbol{t}$, where $\boldsymbol{n} \cdot \boldsymbol{t}=0$. The tangential force leads to a torque like rolling and torsion do, see below.

\subsubsection{Linear contact model}

The simplest normal contact force model, which takes care of excluded volume, and thus the particle elasticity and stiffness, as well as dissipation, involves a linear repulsive and a linear viscous (velocity-dependent) force

$f^{n}=k \delta+\gamma_{0} v_{n}$,

with a spring stiffness $k$, a viscous damping $\gamma_{0}$, and the relative velocity in normal direction $v_{n}=-\boldsymbol{v}_{i j} \cdot \boldsymbol{n}=-\left(\boldsymbol{v}_{i}-\right.$ $\left.\boldsymbol{v}_{j}\right) \cdot \boldsymbol{n}=\dot{\delta}$. This so-called linear spring dashpot (LSD) model describes particle contacts as damped harmonic oscillators, for which the half-period of a vibration — around an equilibrium position with a certain contact force-can be computed analytically [39]. The typical response time, i.e. contact duration, is

$t_{c}=\frac{\pi}{\omega}, \quad$ with $\omega=\sqrt{\left(k / m_{12}\right)-\eta_{0}^{2}}$,

the eigenfrequency of the contact, the rescaled damping coefficient $\eta_{0}=\gamma_{0} /\left(2 m_{i j}\right)$, and the reduced mass $m_{i j}=m_{i} m_{j} /$ $\left(m_{i}+m_{j}\right)$. From the solution of the equation of a half period of the oscillation, one also obtains the coefficient of restitution as the ratio between final (primed) and initial velocity,

$r=v_{n}^{\prime} / v_{n}=\exp \left(-\pi \eta_{0} / \omega\right)=\exp \left(-\eta_{0} t_{c}\right)$.

The contact duration in Eq. (4) is also of practical technical importance, since the integration of the equations of motion is stable only if the integration time-step $\Delta t_{\mathrm{MD}}$ is much smaller than $t_{c}$. Note that $t_{c}$ depends on the magnitude of dissipation: In the extreme case of an overdamped spring, $t_{c}$ can become very large (which would render the contact behavior artificial [47]). Thus, the use of neither too weak nor too strong dissipation is recommended; restitution coefficients between about 0.4 and 0.8 can be seen as "strong" dissipation. Lower values lead to artificially strong viscous 
effects, while larger values correspond to weaker and weaker dissipation, with $r=1$, the elastic limit.

\subsubsection{Adhesive, elasto-plastic contact model}

Here, a variant of the linear hysteretic spring model $[39,87$, 98] is introduced. This model is the simpler version of more complicated nonlinear-hysteretic force laws [71,87,88,98, 99]. The adhesive, plastic (hysteretic) force is

$$
f^{\text {hys }}= \begin{cases}k_{1} \delta & \text { if } k_{2}\left(\delta-\delta_{0}\right) \geq k_{1} \delta \\ k_{2}\left(\delta-\delta_{0}\right) & \text { if } k_{1} \delta>k_{2}\left(\delta-\delta_{0}\right)>-k_{c} \delta \\ -k_{c} \delta & \text { if }-k_{c} \delta \geq k_{2}\left(\delta-\delta_{0}\right)\end{cases}
$$

with $k_{1} \leq k_{2} \leq \hat{k}_{2}$, see Fig. 1 . The lines with slopes $k_{1}$ and $-k_{c}$ define the range of possible force values. Between these two extremes, unloading and reloading follow a line with slope $k_{2}$, which interpolates between $k_{1}$ and a maximum stiffness $\hat{k}_{2}$. Possible equilibrium states are indicated as circles in Fig. 1, where the upper and lower circle correspond to a pre-stressed and stress-free state, respectively. Small perturbations lead, in general, to small deviations along the line with slope $k_{2}$ as indicated by the arrows in Fig. 1 .

During initial loading the force increases linearly with the overlap $\delta$, until the maximum overlap $\delta_{\max }$ is reached $\left(\delta_{\max }\right.$ is kept in memory as a history variable). The line with slope $k_{1}$ thus defines the maximum force possible for a given $\delta$.

During unloading the force drops on a line with slope $k_{2}$, which depends, in general, on $\delta_{\max }$, see Eq. (8). The force at $\delta=\delta_{\text {max }}$ decreases to zero, at overlap $\delta_{0}=\left(1-k_{1} / k_{2}\right) \delta_{\max }$, which resembles the plastic contact deformation. Reloading at any instant leads to an increase of the force along the same line with slope $k_{2}$, until the maximum force is reached; for still increasing $\delta$, the force follows again the line with slope $k_{1}$ and $\delta_{\max }$ has to be adjusted accordingly.

Unloading below $\delta_{0}$ leads to attractive adhesion forces until the minimum force $-k_{c} \delta_{\min }$ is reached at the overlap $\delta_{\min }=\left(k_{2}-k_{1}\right) \delta_{\max } /\left(k_{2}+k_{c}\right)$, a function of the model

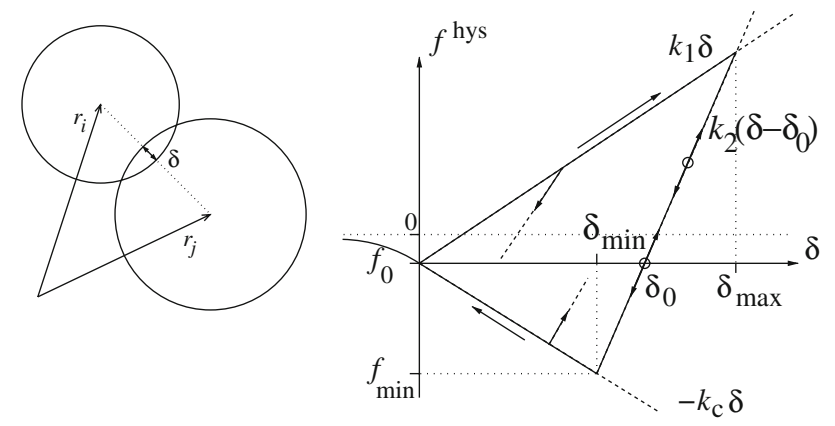

Fig. 1 Left: Two particle contact with overlap $\delta$ in normal direction. Right: Schematic graph of the piece-wise linear, hysteretic, adhesive force-displacement model in normal direction. The non-contact forces, indicated by $f_{0}$ and the line for negative $\delta$, are neglected in the rest of the paper parameters $k_{1}, k_{2}, k_{c}$, and the history parameter $\delta_{\max }$. Further unloading leads to attractive forces $f^{\text {hys }}=-k_{c} \delta$ on the adhesive branch with slope $-k_{c}$. The highest possible attractive force, for given $k_{1}$ and $k_{2}$, is reached for $k_{c} \rightarrow \infty$, so that one has $f_{\min } \geq-\left(k_{2}-k_{1}\right) \delta_{\max }$ for arbitrary $k_{c}$.

A non-linear un-/re-loading behavior would be more realistic, however, due to a lack of detailed experimental informations, the piece-wise linear model is used as a compromise. One reasonable refinement, which accounts for an increasing stiffness with deformation, is a $k_{2}$ value dependent on the maximum overlap. This also implies relatively small and large plastic deformations for weak and strong contact forces, respectively. Unless a constant $k_{2}=\hat{k}_{2}$ is used, the contact model $[43,44,50]$, requires an additional quantity, i.e., the plastic flow limit overlap

$\delta_{\max }^{*}=\frac{\hat{k}_{2}}{\hat{k}_{2}-k_{1}} \phi_{f} \frac{2 a_{1} a_{2}}{a_{1}+a_{2}}$

with the dimensionless plasticity depth, $\phi_{f}$, defined relative to the reduced radius. If the overlap is larger than a fraction $\phi_{f}$ of the particle radius (for $a_{1}=a_{2}$ ), the (maximal) constant stiffness $\hat{k}_{2}$ is used. For different particle radii, the reduced radius increases towards the diameter of the smaller particles in the extreme case of particle-wall contacts (where the wallradius is assumed infinite). This formulation is equivalent to earlier versions $[43,44]$ for almost equal-sized particles, but has some advantages for large size-differences.

Note that a limit stiffness $\hat{k}_{2} \geq k_{2}$ is desirable for practical reasons. If $k_{2}$ would not be limited, the contact duration could become very small so that the time step would have to be reduced below reasonable values. For overlaps smaller than $\delta_{\max }^{*}$, the function $k_{2}\left(\delta_{\max }\right)$ interpolates linearly between $k_{1}$ and $\hat{k}_{2}$ :

$k_{2}:=k_{2}\left(\delta_{\max }\right)=\left\{\begin{array}{ll}\hat{k}_{2} & \text { if } \delta_{\max } \geq \delta_{\max }^{*} \\ k_{1}+\left(\hat{k}_{2}-k_{1}\right) \frac{\delta_{\max }}{\delta_{\max }^{*}} & \text { if } \delta_{\max }<\delta_{\max }^{*}\end{array}\right.$.

While in the case of collisions of particles with large relative velocities - and thus large deformations-dissipation takes place due to the hysteretic nature of the force-law, reasonably strong dissipation of small amplitude deformations is achieved by adding the viscous, velocity dependent dissipative force from Eq. (3) to the hysteretic force, such that $f^{n}=f^{\text {hys }}+\gamma_{0} v_{n}$.

In summary, the adhesive, plastic, hysteretic normal contact model contains the five parameters $k_{1}, \hat{k}_{2}, k_{c}, \phi_{f}$, and $\gamma_{0}$ that respectively account for (i) loading- and (ii) reloadingstiffness and plastic deformation, (iii) adhesion strength, (iv) plastic overlap-range of the model, and (v) viscous dissipation. Finally, we remark that the hysteretic model contains the linear contact model as special case $k_{1} / \hat{k}_{2}=1$ for which $k_{c}$ and $\phi_{f}$ become meaningless. 
Normal van der Waals type particle interactions that lead to attractive forces, see $f_{0}$ in Fig. 1, already when the particles are still separated are not discussed here, for details see [43] and references therein.

\subsection{Tangential contact force laws}

For the tangential degrees of freedom, there are three different force- and torque-laws to be implemented: (i) friction, (ii) rolling resistance, and (iii) torsion resistance.

\subsubsection{Sliding}

For dynamic (sliding) and static friction, the relative tangential velocity of the contact points,

$\boldsymbol{v}_{t}=\boldsymbol{v}_{i j}-\boldsymbol{n}\left(\boldsymbol{n} \cdot \boldsymbol{v}_{i j}\right)$,

is to be considered for the force and torque computations in subsection 2.5 , with the total relative velocity of the particle surfaces at the contact

$\boldsymbol{v}_{i j}=\boldsymbol{v}_{i}-\boldsymbol{v}_{j}+a_{i}^{\prime} \boldsymbol{n} \times \boldsymbol{\omega}_{i}+a_{j}^{\prime} \boldsymbol{n} \times \boldsymbol{\omega}_{j}$,

with the corrected radius relative to the contact point $a_{\alpha}^{\prime}=$ $a_{\alpha}-\delta / 2$, for $\alpha=i, j$. Tangential forces acting on the contacting particles are computed from the accumulated sliding of the contact points along each other, as described in detail in subsection 2.5.1.

\subsubsection{Objectivity}

In general, two particles can rotate together, due to both a rotation of the reference frame or a non-central "collision". The angular velocity $\omega_{0}=\omega_{0}^{n}+\omega_{0}^{t}$, of the rotating reference has the tangential-plane component

$\boldsymbol{\omega}_{0}^{t}=\frac{\boldsymbol{n} \times\left(\boldsymbol{v}_{i}-\boldsymbol{v}_{j}\right)}{a_{i}^{\prime}+a_{j}^{\prime}}$,

which is related to the relative velocity, while the normal component, $\omega_{0}^{n}$, is not. Inserting $\omega_{i}=\omega_{j}=\omega_{0}^{t}$, from Eq. (11), into Eq. (10) leads to zero sliding velocity, proving that the above relations are objective. Tangential forces and torques due to sliding can become active only when the particles are rotating with respect to the common rotating reference frame.

Since action should be equal to reaction, the tangential forces are equally strong, but opposite, i.e., $f_{j}^{t}=-f_{i}^{t}$, while the corresponding torques are parallel but not necessarily equal in magnitude: $\boldsymbol{q}_{i}^{\text {friction }}=-a_{i}^{\prime} \boldsymbol{n} \times \boldsymbol{f}_{i}$, and

\footnotetext{
${ }^{1}$ For rolling and torsion, there is no similar relation between rotational and tangential degrees of freedom: for any rotating reference frame, torques due to rolling and torsion can become active only due to rotation relative to the common reference frame, see below.
}

$\boldsymbol{q}_{j}^{\text {friction }}=\left(a_{j}^{\prime} / a_{i}^{\prime}\right) \boldsymbol{q}_{i}^{\text {friction }}$. Note that tangential forces and torques together conserve the total angular momentum about the pair center of mass

$\boldsymbol{L}_{i j}=\boldsymbol{L}_{i}+\boldsymbol{L}_{j}+m_{i} r_{i \mathrm{~cm}}^{2} \boldsymbol{\omega}_{0}^{t}+m_{j} r_{j \mathrm{~cm}}^{2} \boldsymbol{\omega}_{0}^{t}$,

with the rotational contributions $\boldsymbol{L}_{\alpha}=I_{\alpha} \boldsymbol{\omega}_{\alpha}$, for $\alpha=i, j$, and the distances $r_{\alpha \mathrm{cm}}=\left|\boldsymbol{r}_{\alpha}-r_{\mathrm{cm}}\right|$ from the particle centers to the center of mass $\boldsymbol{r}_{\mathrm{cm}}=\left(m_{i} \boldsymbol{r}_{i}+m_{j} \boldsymbol{r}_{j}\right) /\left(m_{i}+m_{j}\right)$, see Ref. [39]. The change of angular momentum consists of the change of particle spins (first term) and of the change of the angular momentum of the two masses rotating about their common center of mass (second term):

$$
\frac{d \boldsymbol{L}_{i j}}{d t}=\boldsymbol{q}_{i}^{\text {friction }}\left(1+\frac{a_{j}^{\prime}}{a_{i}^{\prime}}\right)+\left(m_{i} r_{i \mathrm{~cm}}^{2}+m_{j} r_{j \mathrm{~cm}}^{2}\right) \frac{d \boldsymbol{\omega}_{0}^{t}}{d t},
$$

which both contribute, but exactly cancel each other, since

$$
\begin{aligned}
\boldsymbol{q}_{i}^{\text {friction }}\left(1+\frac{a_{j}^{\prime}}{a_{i}^{\prime}}\right) & =-\left(a_{i}^{\prime}+a_{j}^{\prime}\right) \boldsymbol{n} \times \boldsymbol{f}_{i} \\
& =-\left(m_{i} r_{i \mathrm{~cm}}^{2}+m_{j} r_{j \mathrm{~cm}}^{2}\right) \frac{d \boldsymbol{\omega}_{0}^{t}}{d t},
\end{aligned}
$$

see [43] for more details.

\subsubsection{Rolling}

A rolling velocity $\boldsymbol{v}_{r}^{0}=-a_{i}^{\prime} \boldsymbol{n} \times \boldsymbol{\omega}_{i}+a_{j}^{\prime} \boldsymbol{n} \times \boldsymbol{\omega}_{j}$, defined in analogy to the sliding velocity, is not objective in general $[17,43]$ — only in the special cases of (i) equal-sized particles or (ii) for a particle rolling on a fixed flat surface.

The rolling velocity should quantify the distance the two surfaces roll over each other (without sliding). Therefore, it is equal for both particles by definition. An objective rolling velocity is obtained by using the reduced radius, $a_{i j}^{\prime}=a_{i}^{\prime} a_{j}^{\prime} /$ $\left(a_{i}^{\prime}+a_{j}^{\prime}\right)$, so that

$\boldsymbol{v}_{r}=-a_{i j}^{\prime}\left(\boldsymbol{n} \times \boldsymbol{\omega}_{i}-\boldsymbol{n} \times \boldsymbol{\omega}_{j}\right)$.

This definition is objective since any common rotation of the two particles vanishes by construction. A more detailed discussion of this issue is beyond the scope of this paper, rather see $[17,43]$ and the references therein.

A rolling velocity will activate torques, acting against the rolling motion, e.g., when two particles are rotating antiparallel with spins in the tangential plane. These torques are then equal in magnitude and opposite in direction, i.e., $\boldsymbol{q}_{i}^{\text {rolling }}=-\boldsymbol{q}_{j}^{\text {rolling }}=a_{i j} \boldsymbol{n} \times \boldsymbol{f}_{r}$, with the quasi-force $\boldsymbol{f}_{r}$, computed in analogy to the friction force, as function of the rolling velocity $\boldsymbol{v}_{r}$ in Sect. 2.5.2; the quasi-forces for both particles are equal and do not act on the centers of mass. Therefore, the total momenta (translational and angular) are conserved. 


\subsubsection{Torsion}

For torsion resistance, the relative spin along the normal direction

$\boldsymbol{v}_{o}=a_{i j}\left(\boldsymbol{n} \cdot \boldsymbol{\omega}_{i}-\boldsymbol{n} \cdot \boldsymbol{\omega}_{j}\right) \boldsymbol{n}$,

is to be considered, which activates torques when two particles are rotating anti-parallel with spins parallel to the normal direction. Torsion is not activated by a common rotation of the particles around the normal direction $\boldsymbol{n} \cdot \boldsymbol{\omega}_{0}=$ $\boldsymbol{n} \cdot\left(\boldsymbol{\omega}_{i}+\boldsymbol{\omega}_{j}\right) / 2$, which makes the torsion resistance objective.

The torsion torques are equal in magnitude and directed in opposite directions, i.e., $\boldsymbol{q}_{i}^{\text {torsion }}=-\boldsymbol{q}_{j}^{\text {torsion }}=a_{i j} \boldsymbol{f}_{o}$, with the quasi-force $f_{o}$, computed from the torsion velocity in Sect. 2.5.3, and also not changing the translational momentum. Like for rolling, the torsion torques conserve the total angular momentum.

\subsubsection{Summary}

The implementation of the tangential force computations for $\boldsymbol{f}_{t}, \boldsymbol{f}_{r}$, and $\boldsymbol{f}_{o}$ as based on $\boldsymbol{v}_{t}, \boldsymbol{v}_{r}$, and $\boldsymbol{v}_{o}$, respectively, is assumed to be identical, i.e., even the same subroutine is used, but with different parameters as specified below. The difference is that friction leads to a force in the tangential plane (changing both translational and angular momentum), while rolling- and torsion-resistance lead to quasi-forces in the tangential plane and the normal direction, respectively, changing the particles' angular momentum only.

For more details on tangential contact models, friction, rolling and torsion, see Refs. [2,16,17,43,44].

\subsection{The tangential contact model}

The tangential contact model presented now is a single procedure (subroutine) that can be used to compute either sliding, rolling, or torsion resistance. The subroutine needs a relative velocity as input and returns the respective force or quasiforce as function of the accumulated deformation. The sliding/sticking friction model will be introduced in detail, while rolling and torsion resistance are discussed where different.

\subsubsection{Sliding/sticking friction model}

The tangential force is coupled to the normal force via Coulomb's law, $f^{t} \leq f_{C}^{s}:=\mu^{s} f^{n}$, where for the sliding case one has dynamic friction with $f^{t}=f_{C}^{t}:=\mu^{d} f^{n}$. The dynamic and the static friction coefficients follow, in general, the relation $\mu^{d} \leq \mu^{s}$. The static situation requires an elastic spring in order to allow for a restoring force, i.e., a non-zero remaining tangential force in static equilibrium due to activated Coulomb friction.
If a purely repulsive contact is established, $f^{n}>0$, the tangential force can be active. For an adhesive contact, Coulombs law has to be modified in so far that $f^{n}$ is replaced by $f^{n}+k_{c} \delta$. In this model, the reference for a contact is no longer the zero force level, but the adhesive, attractive force level along $-k_{c} \delta$.

If a contact is active, one has to project (or better rotate) the tangential spring into the actual tangential plane, since the frame of reference of the contact may have rotated since the last time-step. The tangential spring

$\xi=\xi^{\prime}-n\left(n \cdot \xi^{\prime}\right)$,

is used for the actual computation, where $\xi^{\prime}$ is the old spring from the last iteration, with $|\xi|=\left|\xi^{\prime}\right|$ enforced by appropriate scaling/rotation. If the spring is new, the tangential springlength is zero, but its change is well defined after the first, initiation step.

In order to compute the changes of the tangential spring, a tangential test-force is first computed as the sum of the tangential spring force and a tangential viscous force (in analogy to the normal viscous force)

$\boldsymbol{f}_{0}^{t}=-k_{t} \boldsymbol{\xi}-\gamma_{t} \boldsymbol{v}_{t}$

with the tangential spring stiffness $k_{t}$, the tangential dissipation parameter $\gamma_{t}$, and $\boldsymbol{v}_{t}$ from Eq. (9). As long as $\left|\boldsymbol{f}_{0}^{t}\right| \leq f_{C}^{s}$, with $f_{C}^{s}=\mu^{s}\left(f^{n}+k_{c} \delta\right)$, one has static friction and, on the other hand, for $\left|\boldsymbol{f}_{0}^{t}\right|>f_{C}^{s}$, sliding friction becomes active. As soon as $\left|\boldsymbol{f}_{0}^{t}\right|$ gets smaller than $f_{C}^{d}$, static friction becomes active again.

In the static friction case, below the Coulomb limit, the tangential spring is incremented

$\xi^{\prime}=\xi+v_{t} \Delta t_{\mathrm{MD}}$,

to be used in the next iteration in Eq. (17), and the tangential force $\boldsymbol{f}^{t}=\boldsymbol{f}_{0}^{t}$ from Eq. (18) is used. In the sliding friction case, the tangential spring is adjusted to a length consistent with Coulombs condition, so that

$\xi^{\prime}=-\frac{1}{k_{t}}\left(f_{C}^{d} \boldsymbol{t}+\gamma_{t} \boldsymbol{v}_{t}\right)$

with the tangential unit vector, $\boldsymbol{t}=\boldsymbol{f}_{0}^{t} /\left|\boldsymbol{f}_{0}^{t}\right|$, defined by Eq. (18), and thus the magnitude of the Coulomb force is used. Inserting $\xi^{\prime}$ from Eq. (20) into Eq. (18) during the next iteration will lead to $\boldsymbol{f}_{0}^{t} \approx f_{C}^{d} \boldsymbol{t}$.

Note that $\boldsymbol{f}_{0}^{t}$ and $\boldsymbol{v}_{t}$ are not necessarily parallel in three dimensions. However, the mapping in Eq. (20) works always, rotating the new spring such that the direction of the frictional force is unchanged and, at the same time, limiting the spring in length according to Coulombs law. In short notation the tangential contact law reads

$\boldsymbol{f}^{t}=f^{t} \boldsymbol{t}=+\min \left(f_{C},\left|\boldsymbol{f}_{0}^{t}\right|\right) \boldsymbol{t}$, 
where $f_{C}$ follows the static/dynamic selection rules described above. The torque on a particle due to frictional forces at this contact is $q^{\text {friction }}=\boldsymbol{l}_{i}^{c} \times \boldsymbol{f}_{i}^{c}$, where $\boldsymbol{l}_{i}^{c}$ is the branch vector connecting the center of the particle with the contact point. Note that the torque on the contact partner is generally different in magnitude, since $\boldsymbol{l}_{i}^{c}$ can be different, but points in the same direction; see Sect. 2.4.2 for details on this.

The four parameters for the friction law are $k_{t}, \mu_{s}, \phi_{d}=$ $\mu_{d} / \mu_{s}$, and $\gamma_{t}$, accounting for tangential stiffness, the static friction coefficient, the dynamic friction ratio, and the tangential viscosity, respectively. Note that the tangential force described above is identical to the classical Cundall-Strack spring only in the limits $\mu=\mu^{s}=\mu^{d}$, i.e., $\phi_{d}=1$, and $\gamma_{t}=0$. The sequence of computations and the definitions and mappings into the tangential direction can be used in 3D as well as in $2 \mathrm{D}$.

\subsubsection{Rolling resistance model}

The three new parameters for rolling resistance are $k_{r}, \mu_{r}$, and $\gamma_{r}$, while $\phi_{r}=\phi_{d}$ is used from the friction law. The new parameters account for rolling stiffness, a static rolling "friction" coefficient, and rolling viscosity, respectively. In the subroutine called, the rolling velocity $\boldsymbol{v}_{r}$ is used instead of $\boldsymbol{v}_{t}$ and the computed quasi-force $\boldsymbol{f}_{r}$ is used to compute the torques, $\boldsymbol{q}^{\text {rolling }}$, on the particles.

\subsubsection{Torsion resistance model}

The three new parameters for rolling resistance are $k_{o}, \mu_{o}$, and $\gamma_{o}$, while $\phi_{o}=\phi_{d}$ is used from the friction law. The new parameters account for torsion stiffness, a static torsion "friction" coefficient, and torsion viscosity, respectively. In the subroutine, the torsion velocity $\boldsymbol{v}_{o}$ is used instead of $\boldsymbol{v}_{t}$ and the projection is a projection along the normal unit-vector, not into the tangential plane as for the other two models. The computed quasi-force $f_{o}$ is then used to compute the torques, $\boldsymbol{q}^{\text {torsion }}$, on the particles.

\subsection{Background friction}

Note that the viscous dissipation takes place in a two-particle contact. In the bulk material, where many particles are in contact with each other, this dissipation mode is very inefficient for long-wavelength cooperative modes of motion [47,48]. Therefore, an additional damping with the background can be introduced, so that the total force on particle $i$ is

$\boldsymbol{f}_{i}=\sum_{j}\left(f^{n} \boldsymbol{n}+f^{t} \boldsymbol{t}\right)-\gamma_{b} \boldsymbol{v}_{i}$
Table 1 The microscopic contact model parameters

\begin{tabular}{ll}
\hline Property & Symbol \\
\hline Time unit & $t_{u}$ \\
Length unit & $x_{u}$ \\
Mass unit & $m_{u}$ \\
Particle radius & $a_{0}$ \\
Material density & $\rho$ \\
Elastic stiffness (variable) & $k_{2}$ \\
Maximal elastic stiffness (constant) & $k=\hat{k}_{2}$ \\
Plastic stiffness & $k_{1} / k$ \\
Adhesion "stiffness" & $k_{c} / k$ \\
Friction stiffness & $k_{t} / k$ \\
Rolling stiffness & $k_{r} / k$ \\
Torsion stiffness & $k_{o} / k$ \\
Plasticity depth & $\phi_{f}$ \\
Coulomb friction coefficient & $\mu=\mu_{d}=\mu_{s}$ \\
Dynamic to static friction ratio & $\phi_{d}=\mu_{d} / \mu_{s}$ \\
Rolling "friction" coefficient & $\mu_{r}$ \\
Torsion "friction" coefficient & $\mu_{o}$ \\
Normal viscosity & $\gamma=\gamma_{n}$ \\
Friction viscosity & $\gamma_{t} / \gamma$ \\
Rolling viscosity & $\gamma_{r} / \gamma$ \\
Torsion viscosity & $\gamma_{o} / \gamma$ \\
Background viscosity & $\gamma_{b} / \gamma$ \\
Background viscous torque & $\gamma_{b r} / \gamma$ \\
\hline &
\end{tabular}

and the total torque

$\boldsymbol{q}_{i}=\sum_{j}\left(\boldsymbol{q}^{\text {friction }}+\boldsymbol{q}^{\text {rolling }}+\boldsymbol{q}^{\text {torsion }}\right)-\gamma_{b r} a_{i}^{2} \boldsymbol{\omega}_{i}$,

with the damping artificially enhanced in the spirit of a rapid relaxation and equilibration. The sum in Eqs. (22) and (23) takes into account all contact partners $j$ of particle $i$, but the background dissipation can be attributed to the medium between the particles. Note that the effect of $\gamma_{b}$ and $\gamma_{b r}$ should be checked for each set of parameters: it should be small in order to exclude artificial over-damping.

The set of parameters is summarized in Table 1. Note that only a few parameters are specified with dimensions, while the other paramters are expressed as ratios.

\section{Tension test simulation results}

In this section, uni-axial tension tests and a few compression tests are presented. The tests consists of three stages: (i) pressure sintering, (ii) stress-relaxation, and (iii) the compressionor tension-test itself. The contact parameters, as introduced in the previous section, are summarized in Table 1 and 
typical values are given in Table 2. These parameters are used for particle-particle contacts, the same for all stages, unless explicitly specified.

For pressure sintering, a loose assembly of particles is first compressed with an isotropic stress $p_{s} 2 a / \hat{k}_{2} \approx 0.02$ in a cuboid volume. The adhesive contact forces are activated this way. Two of the six walls are adhesive, with $k_{c}^{\text {wall }} / \hat{k}_{2}=20$, so that the sample sticks to them, while all other walls are adhesionless, so that they can be easily removed in the second stage. (During compression and sintering, the walls could all be without adhesion, since the high pressure used keeps the sample together anyway — only later for relaxation, adhesion must switched on. If not the sample does not remain a solid, and it also could lose contact with the walls, which are later used to apply the tensile strain.)

Note that all walls are frictionless during sintering, while the particles are slightly adhesive and frictional. (If the walls would be frictional, the pressure from a certain wall would not be transferred completely to the respective opposite wall, since frictional forces carry part of the load-an effect that is known since the early work of Janssen $[23,77,86]$.)

Pressure-sintering is stopped when the kinetic energy of the sample is many orders of magnitude smaller than the potential energy - typically ten orders of magnitude.

During stress-relaxation all wall stresses are slowly released to $p_{r} / p_{s} \ll 1$ and the sample is relaxed until the kinetic energy is much smaller than the potential energy. The sample is ready for the tension tests. In fact, the same initial configuration is used for all the tests presented below. Note that the non-adhesive side walls still feel a very small external stress that is not big enough to affect the dynamics of the tension test, it is just convenient to keep the walls close to the sample.

For the tension test wall friction is typically active, but some variation does not show a big effect. One of the sticky walls is slowly and smoothly moved outwards like described and applied in earlier studies [42,45], following a prescribed cosine-function with time.

\subsection{Model parameters}

The system contains $N=1728$ particles with radii $a_{i}$ drawn from a Gaussian distribution around $a=0.005 \mathrm{~mm}[13,14]$. The contact model parameters are summarized in Tables 1 and 2. The volume fraction, $v=\sum_{i} V\left(a_{i}\right) / V$, with the particle volume $V\left(a_{i}\right)=(4 / 3) \pi a_{i}^{3}$, reached during pressure sintering with $2 a p_{s} / \hat{k}_{2}=0.02$ is $v_{s}=0.6754$. The coordination number is $\mathcal{C} \approx 7.16$ in this state. After stress-relaxation, these values have changed to $v \approx 0.629$ and $\mathcal{C} \approx 6.19$. A different preparation procedure (with adhesion $k_{c} / \hat{k}_{2}=0$ during sintering) does not lead to a difference in density after sintering. However, one observes $v \approx 0.630$ and $\mathcal{C} \approx 6.23$
Table 2 Microscopic material parameters used (second column)

\begin{tabular}{|c|c|c|c|}
\hline Symbol & Value & rescaled units & SI-units \\
\hline$t_{u}$ & 1 & $1 \mu \mathrm{s}$ & $10^{-6} \mathrm{~s}$ \\
\hline$x_{u}$ & 1 & $1 \mathrm{~mm}$ & $10^{-3} \mathrm{~m}$ \\
\hline$m_{u}$ & 1 & $1 \mathrm{mg}$ & $10^{-6} \mathrm{~kg}$ \\
\hline$a_{0}$ & 0.005 & $5 \mu \mathrm{m}$ & $5.10^{-6} \mathrm{~m}$ \\
\hline$\rho$ & 2 & $2 \mathrm{mg} / \mathrm{mm}^{3}$ & $2,000 \mathrm{~kg} / \mathrm{m}^{3}$ \\
\hline$k=\hat{k}_{2}$ & 5 & $5 \mathrm{mg} / \mu \mathrm{s}^{2}$ & $5.10^{6} \mathrm{~kg} / \mathrm{s}^{2}$ \\
\hline$k_{1} / k$ & 0.5 & & \\
\hline$k_{c} / k$ & 0.5 & & \\
\hline$k_{t} / k$ & 0.2 & & \\
\hline$k_{r} / k=k_{o} / k$ & 0.1 & & \\
\hline$\phi_{f}$ & 0.05 & & \\
\hline$\mu=\mu_{d}=\mu_{s}$ & 1 & & \\
\hline$\phi_{d}=\mu_{d} / \mu_{s}$ & 1 & & \\
\hline$\mu_{r}=\mu_{o}$ & 0.1 & & \\
\hline$\gamma=\gamma_{n}$ & $5.10^{-5}$ & $5.10^{-5} \mathrm{mg} / \mu \mathrm{s}$ & $5.10^{1} \mathrm{~kg} / \mathrm{s}$ \\
\hline$\gamma_{t} / \gamma$ & 0.2 & & \\
\hline$\gamma_{r} / \gamma=\gamma_{o} / \gamma$ & 0.05 & & \\
\hline$\gamma_{b} / \gamma$ & 4.0 & & \\
\hline$\gamma_{b r} / \gamma$ & 1.0 & & \\
\hline
\end{tabular}

The third column contains these values in the appropriate units, i.e., when the time-, length-, and mass-unit are $\mu \mathrm{s}$, mm, and $\mathrm{mg}$, respectively. Column four contains the parameters in SI-units. Energy, force, acceleration, and stress have to be scaled with factors of $1,10^{3}, 10^{9}$, and $10^{9}$, respectively, for a transition from rescaled to SI-units

after relaxation. For both preparation procedures the tension test results are virtually identical, so that only the first procedure is used in the following.

The material parameters used for the particle contacts are given in Table 2. The particle-wall contact parameters are the same, except for cohesion and friction, for which $k_{c}^{\text {wall }} / \hat{k}_{2}=$ 20 and $\mu^{\text {wall }}=10$ are used - the former during all stages, the latter only during tensile testing.

The choice of numbers and units is such that the particles correspond to spheres with several microns in radius. The magnitude of stiffness $k$ cannot be compared directly with the material bulk modulus $C$, since it is a contact property. However, there are relations from micro-macro transition analysis, which allow to relate $k$ and $C \sim k \mathcal{C} a^{2} / V$ [42].

Using the parameter $k=\hat{k}_{2}$ in Eq. (4) leads to a typical contact duration (half-period) $t_{c} \approx 6.510^{-4} \mu \mathrm{s}$, for a normal collision of a large and a small particle with $\gamma=0$. Accordingly, an integration time-step of $t_{\mathrm{MD}}=5.10^{-6} \mu \mathrm{s}$ is used, in order to allow for a "safe" integration of the equations of motion. Note that not only the normal "eigenfrequency" but also the eigenfrequencies in tangential and rotational direction have to be considered as well as the viscous 

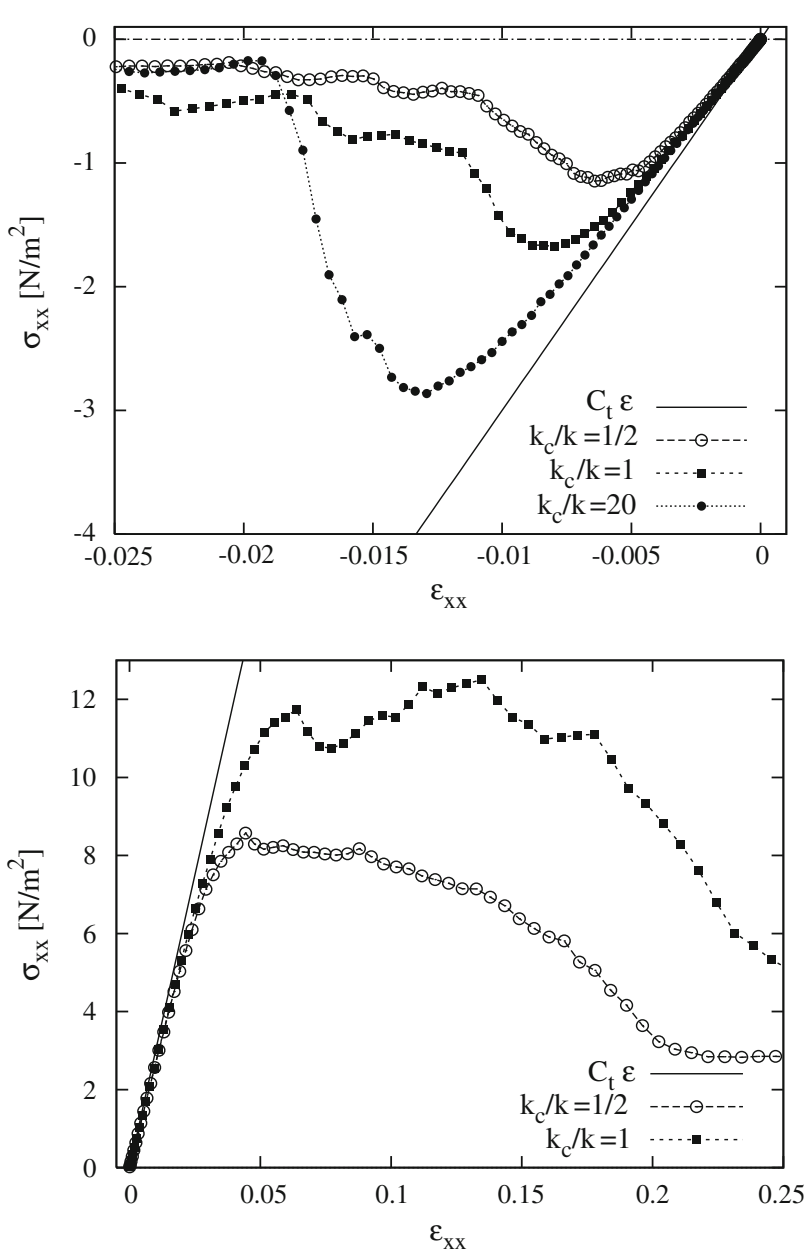

Fig. 2 Top Axial tensile stress plotted against tensile strain for simulations with weak, moderate and strong particle contact adhesion; the $k_{c} / k$ values are given in the inset. The line gives a fit to the linear elastic regime with $C_{t}=3.10^{11} \mathrm{~N} / \mathrm{m}^{2}$. Bottom Axial compressive stress plotted against compressive strain for two of the parameter sets from the top panel. The initial slope is the same as in the top panel, indicating that the linear elastic regime is identical for tension and compression

response times $t_{\gamma} \approx m / \gamma$. All of the physical time-scales should be considerably larger than $t_{\mathrm{MD}}$, whereas the viscous response times should be even larger, so that $t_{\gamma}>t_{c}>t_{\mathrm{MD}}$. A more detailed discussion of all the effects due to the interplay between the model parameters and the related times is, however, far from the scope of this paper.

\subsection{Tensile strength and contact adhesion}

The tensile (compressive) test is performed uni-axially in $x$ direction by increasing (reducing) slowly and smoothly the distance between the two sticky walls. (The same initial sample, prepared with $k_{c} / k=1 / 2$, is used for all tests reported here.) The stress-strain curves for different cohesion are plotted in Fig. 2, for both tension and compression.

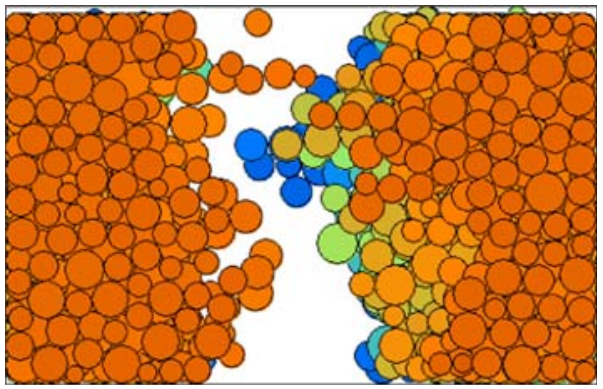

Fig. 3 Color online Snapshot from a tensile test with $k_{c} / k=1 / 2$ at horizontal strain of $\varepsilon_{x x} \approx 0.8$. The color code denotes the distance from the viewer: blue, green, and red correspond to large, moderate, and short distance

The axial tensile stress initially increases linearly with strain, practically independent from the contact adhesion strength. With increasing strain, a considerable number of contacts are opened due to tension - contacts open more easily for smaller adhesion (data not shown). This leads to a decrease of the stress-strain slope, then the stress reaches a maximum and, for larger strain, turns into a softening failure mode. As expected, the maximal stress is increasing with contact adhesion $k_{c} / k$. The compressive strength is 6-7 times larger than the tensile strength, and a larger adhesion force also allows for larger deformation before failure. The sample with weakest adhesion, $k_{c} / k=1 / 2$, shows tensile and compressive failure at strains $\varepsilon_{x x} \approx-0.006$ and $\varepsilon_{x x} \approx 0.045$, respectively.

Note that for tension, the post-peak behavior for the test with $k_{c} / k=20$ is different from the other two cases, due to the strong particle-particle contact adhesion. In this case, the tensile fracture occurs at the wall (except for a few particles that remain in contact with the wall). This is in contrast to the other two cases with smaller bulk-adhesion, where the fracture occurs in the bulk, see Fig. 3.

\subsection{Tensile strength and friction}

In Fig. 4, the rather weak effect of various values of friction, rolling- and torsion-resistance becomes evident. For the tensile tests presented here, even the largest friction, rolling- and torsion-resistance used $\mu=\mu_{r}=\mu_{o}=100$ does not lead to a considerable increase of tensile strength. Furthermore, simulations with different static and dynamic friction coefficients, $\mu_{s}=1$ and $\mu_{d}=0.5$, also do not lead to different behavior under tension; they rather show, that the contact model is able to deal with different coefficients.

\section{Conclusion}

The present study reviews many issues related to soft particle force models. As compromise between simplicity and reality, 

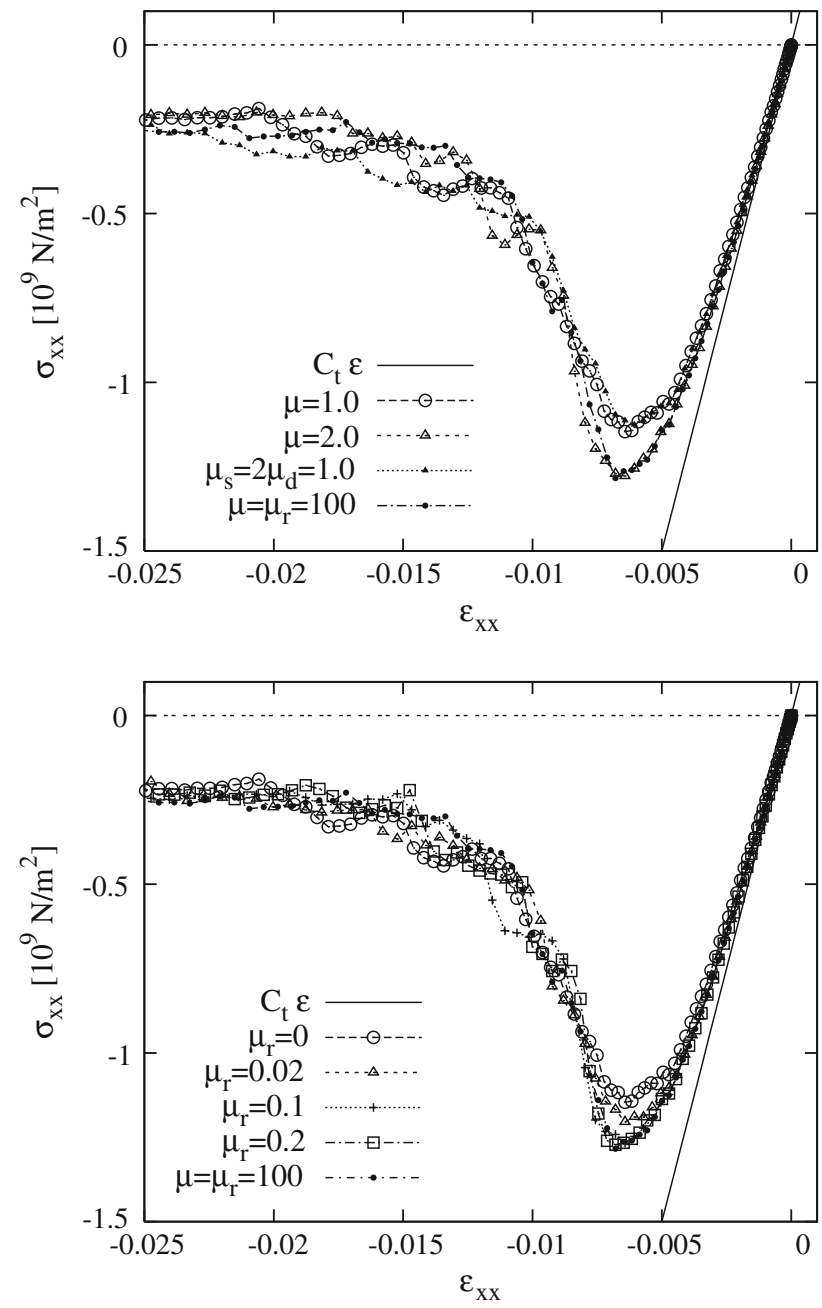

Fig. 4 Tensile stress plotted against tensile strain for simulations with weak contact adhesion $k_{c} / k=1 / 2$, and with different rolling- and torsion friction coefficients, as given in the inset. The lines show the same fit as in Fig. 2

a special contact model is introduced, involving elastic-viscoplastic normal contact forces, adhesion, friction, and rollingas well as torsion resistance-all in one. A set of exemplary

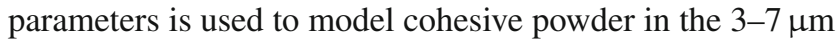
range. The powder-sample is first pressure-sintered, then the walls are removed from the solid cuboid sample, and finally the sample is subjected to uni-axial, strain-controlled tension until it fails. Stronger contact adhesion leads to considerably larger tensile strength, while the effect of rolling- and torsionresistance is very weak for the parameter combinations used here-for related results, see Refs. [43,44,46,51].

The samples are sintered using the force- and torquemodels described in Sect. 2-most parameters are kept constant throughout the three phases of the tensile test, proving that the advanced model is able to mimick a wealth of different behavior without further adjustments. The contact model presented here, besides many model assumptions, still involves a considerable number of parameters. As the tension test has shown, some of them (rolling- and torsionresistance) seem less important for specific physical properties than others. Naturally, contact adhesion is most important for the tensile strength of the material, but also friction shows an effect to be examined further. Note that some important model parameters, like the ratios $k_{1} / k$ and $k_{t} / k$ were not yet studied in detail.

The quantitative tuning of the DEM model to real experimental data remains the challenge for future research. The results presented here have units that were not supposed to exactly mimick a real material, but should be rather close to those of fine powders. Some tuning can be done by rescaling, but a real fine-adjustement will require a more systematic study of all contact model parameters- to be done in the future.

Acknowledgments An early version of this paper was printed as a scientific report of the project "Flow of cohesive fine powders" in the research group "Behavior of Granular Media", sponsored by the Deutsche Forschungsgemeinschaft (DFG). Valuable discussions with the project partners H.-J. Butt, M. Kappl, J. Tomas, and R. Tykhoniuk are acknowledged, as well as advice from A. Suiker, L. Brendel, and S. McNamara, about contact models in all details, and the communication with Danie Els, especially about the objectivity of the model-forces and -torques. Finally, the support from the Stichting voor Fundamenteel Onderzoek der Materie (FOM), financially supported by the Nederlandse Organisatie voor Wetenschappelijk Onderzoek (NWO), is gratefully acknowledged, for sponsoring the related project "Hysteresis and Creep in granular media" in the framework of the FOM-program "Granular Matter".

Open Access This article is distributed under the terms of the Creative Commons Attribution Noncommercial License which permits any noncommercial use, distribution, and reproduction in any medium, provided the original author(s) and source are credited.

\section{References}

1. Allen, M.P., Tildesley, D.J.: Computer Simulation of Liquids. Oxford University Press, Oxford (1987)

2. Bartels, G., Unger, T., Kadau, D., Wolf, D.E., Kertesz, J.: The effect of contact torques on porosity of cohesive powders. Granular Matter 7, 139 (2005)

3. Bashir, Y.M., Goddard, J.D.: A novel simulation method for the quasi-static mechanics of granular assemblages. J Rheol 35(5), 849-885 (1991)

4. Berger, F.: Das Gesetz des Kraftverlaufes beim Stoß. Friedr. Vieweg \& Sohn AG (1924)

5. Brendel, L.: Modeling of caked contacts in DEMs. Chem Eng Technol 29(11), 1355-1359 (2006)

6. Brendel, L., Dippel, S.: Lasting contacts in molecular dynamics simulations. In: Herrmann, H.J., Hovi, J.P., Luding, S. (eds) Physics of Dry Granular Media. Kluwer, Dordrecht, p 313 (1998)

7. Brilliantov, N.V., Spahn, F., Hertzsch, J.M., Pöschel, T.: Model for collisions in granular gases. Phys Rev E 53(5), 5382 (1996)

8. Butt, H.J., Cappella, B., Kappl, M.: Force measurements with the atomic force microscope: Technique, interpretation and applications. Surf. Sci. Rep. 59(1-6), 1-152 (2005) 
9. Castellanos, A.: The relationship between attractive interparticle forces and bulk behavior in dry and uncharged fine powders. Adv. Phys. 54(4), 263-376 (2005)

10. Coste, C., Falcon, E., Fauve, S.: Propagations d'ondes nonlinéaires dans une chaîne de bille s en contact de Hertz. In: Petit C., Pijaudier-Cabot G., Reynouard J.M. (eds) Des géomatériaux aux ouvrages: expérimentations et modélis ations, Hermes, Paris, pp 33-52 (in french, 1995)

11. Coste, C., Falcon, E., Fauve, S.: Solitary waves in a chain of beads under Hertz contact. Phys. Rev. E 56(5), 6104-6117 (1997)

12. Cundall, P.A., Strack, O.D.L.: A discrete numerical model for granular assemblies. Géotechnique 29(1), 47-65 (1979)

13. David, C.T., Rojo, R.G., Herrmann, H.J., Luding, S.: Hysteresis and creep in powders and grains. In: Garcia-Rojo, R., Herrmann, H.J., McNamara, S. (eds) Powders and Grains 2005, Balkema, Leiden, Netherlands, pp. 291-294 (2005)

14. David, C.T., Garcia-Rojo, R., Herrmann, H.J., Luding, S.: Powder flow testing with $2 \mathrm{~d}$ and $3 \mathrm{~d}$ biaxial and triaxial simulations. Particle Particle Syst. Charact. 24(1), 29-33 (2007)

15. Derjaguin, B.V., Muller, V.M., Toporov, Y.P.: Effect of contact deformation on adhesion of particles. J. Colloid Interf. Sci. 53, 314-326 (1975)

16. Dintwa, E., van Zeebroeck, M., Tijskens, E., Ramon, H.: Torsion of viscoelastic spheres in contact. Granular Matter 7(2-3), 169179 (2005)

17. Els, D.: Definition of roll velocity for spherical particles. Granular Matter (2006, submitted)

18. Foerster, S.F., Louge, M.Y., Chang, H., Allia, K.: Measurements of the collision properties of small spheres. Phys. Fluids 6(3), 11081115 (1994)

19. Grof, Z., Lawrence, C.J., Stepanek, F.: Computer simulation of evolving capillary bridges in granular media. Granular Matter 10(2), 93-103 (2008)

20. Heim, L.O., Butt, H.J., Blum, J., Schrapler, R.: A new method for the analysis of compaction processes in high-porosity agglomerates. Granular Matter 10(2), 89-91 (2008)

21. Herrmann, H.J., Hovi, J.P., Luding, S.: (eds) Physics of dry granular media-NATO ASI Series E 350. Kluwer, Dordrecht (1998)

22. Hertz, H.: Über die Berührung fester elastischer Körper. J für Die Reine U Angew Math 92, 136 (1882)

23. Janssen, H.A.: Versuche über Getreidedruck in Silozellen. Zeitschr d Vereines Deutscher Ingenieure 39(35), 1045-1049 (1895)

24. Jenkins, J.T., Koenders, M.A.: Hydrodynamic interaction of rough spheres. Granular Matter 7(1), 13-18 (2005)

25. Johnson, K.L.: Contact Mechanics. Cambridge University Press, Cambridge (1989)

26. Johnson, K.L., Kendall, K., Roberts, A.D.: Surface energy and contact of elastic solids. Proc R Soc Lond Ser A 324 (1558), 301 (1971)

27. Johnson, P.C., Jackson, R.: Frictional-collisional constitutive relations for granular materials, with application to plane shearing. $\mathrm{J}$ Fluid Mech 176, 67 (1987)

28. Kadau, D., Schwesig, D., Theuerkauf, J., Wolf, D.E.: Influence of particle elasticity in shear testers. Granular Matter 8, 34-40 (2006)

29. Kafui, K.D., Thornton, C.: Numerical simulations of impact breakage of spherical crystalline agglomerate. Powder Technol 109, 113-132 (2000)

30. Kappl, M., Heim, L., Butt, H.J., Luding, S., Tykhoniuk, R., Tomas, J.: From grains to powders: from single particle contact mechanics measurements to bulk powder properties. In: Garcia-Rojo, R., Herrmann, H.J., McNamara, S. (eds) Powders and Grains 2005, Balkema, Leiden, Netherlands, pp. 493-497 (2005)

31. Kun, F., Herrmann, H.J.: Damage development under gradual loading of composites. J. Mater. Sci. 35(18), 4685-4693 (2000)
32. Kuwabara, G., Kono, K.: Restitution coefficient in a collision between two spheres. Jpn. J. Appl. Phys. 26(8), 1230-1233 (1987)

33. Labous, L., Rosato, A.D., Dave, R.: Measurements of collision properties of spheres using high-speed video analysis. Phys. Rev. E 56, 5715 (1997)

34. Lätzel, M., Luding, S., Herrmann, H.J., Howell, D.W., Behringer, R.P.: Comparing simulation and experiment of a $2 \mathrm{~d}$ granular couette shear device. Eur. Phys. J. Eng. 11(4), 325-333 (2003)

35. Leroy, B.: Collision between two balls accompanied by deformation: A qualitative approach to Hertz's theory. Am. J. Phys. 53(4), 346-349 (1985)

36. Lian, G., Adams, M.J., Thornton, C.: Elastohydrodynamic collisions of solid spheres. J Fluid. Mech. 311, 141 (1996)

37. Lorenz, A., Tuozzolo, C., Louge, M.Y.: Measurements of impact properties of small, nearly spherical particles. Exp. Mech. 37(3), 292-297 (1997)

38. Lubachevsky, B.D.: How to simulate billards and similar systems. J. Comp. Phys. 94(2), 255 (1991)

39. Luding, S.: Collisions and contacts between two particles. In: Herrmann, H.J., Hovi, J.P., Luding, S. (eds) Physics of dry granular media—NATO ASI Series E350. Kluwer, Dordrecht, p. 285 (1998)

40. Luding, S.: Micro-macro models for anisotropic granular media. In: Vermeer, P.A., Ehlers, W., Herrmann, H.J., Ramm, E. (eds) Modelling of cohesive-frictional Materials, Balkema, pp 195-206 (ISBN 041536023 4) (2004a)

41. Luding, S.: Molecular dynamics simulations of granular materials. In: Hinrichsen, H., Wolf, D.E. (eds) The Physics of Granular Media, Wiley VCH, Weinheim, pp. 299-324 (2004b)

42. Luding, S.: Anisotropy in cohesive, frictional granular media. J. Phys. Condens. Matter 17, S2623-S2640 (2005)

43. Luding, S.: About contact force-laws for cohesive frictional materials in $2 \mathrm{~d}$ and 3d. In: Walzel, P., Linz, S., Krülle, C., Grochowski, R. (eds) Behavior of Granular Media, Shaker Verlag, pp 137-147, band 9, Schriftenreihe Mechanische Verfahrenstechnik, ISBN 38322-5524-9 (2006)

44. Luding, S.: Contact models for very loose granular materials. In: Eberhard P. (ed) Symposium on Multiscale Problems in Multibody System Contacts, Springer, Heidelberg, pp. 135-150. ISBN 9781-4020-5980-3 (2007)

45. Luding, S., Herrmann, H.J.: Micro-macro transition for cohesive granular media. In: Diebels S. (Ed.) Bericht Nr. II-7, Inst. für Mechanik, Universität Stuttgart (2001)

46. Luding, S., Suiker, A.: Self-healing of damaged particulate materials through sintering. Philos. Mag. (2008, submitted)

47. Luding, S., Clément, E., Blumen, A., Rajchenbach, J., Duran, $\mathrm{J} .:$ Anomalous energy dissipation in molecular dynamics simulations of grains: The "detachment effect". Phys. Rev. E 50, 4113 (1994)

48. Luding, S., Clément, E., Blumen, A., Rajchenbach, J., Duran, J.: The onset of convection in molecular dynamics simulations of grains. Phys. Rev. E 50, R1762 (1994)

49. Luding, S., Clément, E., Blumen, A., Rajchenbach, J., Duran, J.: Studies of columns of beads under external vibrations. Phys. Rev. E 49(2), 1634 (1994)

50. Luding, S., Manetsberger, K., Muellers, J.: A discrete model for long time sintering. J. Mech. Phys. Solids 53(2), 455-491 (2005)

51. Luding, S., Suiker, A., Kadashevich, I.: Discrete element modeling of self-healing processes in damaged particulate materials. In: Schmets, A.J.M., van der Zwaag, S. (eds) Proceedings of the 1st International Conference on Self Healing Materials, Springer series in Material Science, Berlin, Germany (ISBN 978-1-40206249-0 (2007)

52. Matuttis, H.G., Luding, S., Herrmann, H.J.: Discrete element methods for the simulation of dense packings and heaps made of 
spherical and non-spherical particles. Powder Technol. 109, 278$292(2000)$

53. Mindlin, R.D.: Compliance of elastic bodies in contact. J. Appl. Mech. 16, 259 (1949)

54. Mindlin, R.D., Deresiewicz, H.: Elastic spheres in contact under varying oblique forces. J. Appl. Mech. 20, 327 (1953)

55. Moreau, J.J.: New computation methods in granular dynamics. In: Powders and Grains, vol. 93. Balkema, Rotterdam, p 227 (1993)

56. Moreau, J.J.: Some numerical methods in multibody dynamics: application to granular materials. Eur J Mech A 13, 93 (1994)

57. Oda, M., Iwashita, K.: Study on couple stress and shear band development in granular media based on numerical simulation analyses. Int. J. Eng. Sci. 38, 1713-1740 (2000)

58. Oda, M., Kazama, H.: Microstructure of shear bands and its relation to the mechanism of dilatancy and failure of dense granular soils. Géotechnique 48(4), 465-481 (1998)

59. Oda, M., Iwashita, K., Kazama, H.: Micro-structure developed in shear bands of dense granular soils and its computer simulationmechanism of dilatancy and failure. In: Fleck, N.A., Cocks, A.C.E. (eds) IUTAM Symposium on Mechanics of Granular and Porous Materials. Kluwer, Dordrecht, pp 353-364 (1997)

60. Pao, Y.H.: Extension of the Hertz theory of impact to the viscoelastic case. J. Appl. Phys. 26, 1083 (1955)

61. Pöschel, T., Schwager, T.: Computational Granular Dynamics. Springer, Berlin (2005)

62. Pöschel, T., Schwager, T., Brilliantov, N.V.: Rolling friction of a hard cylinder on a viscous plane. Eur. J. Phys. 10, 169-175 (1999)

63. Radjai, F., Jean, M., Moreau, J.J., Roux, S.: Force distribution in dense two-dimensional granular systems. Phys. Rev. Lett. 77(2), 274 (1996)

64. Radjai, F., Schäfer, J., Dippel, S., Wolf, D.: Collective friction of an array of particles: A crucial test for numerical algorithms. J. Phys. I France 7, 1053 (1997)

65. Radjai, F., Wolf, D.E., Jean, M., Moreau, J.J.: Bimodal character of stress transmission in granular packings. Phys. Rev. Lett. 80(1), 61-64 (1998)

66. Raman, C.V.: The photographic study of impact at minimal velocities. Phys. Rev. 12, 442-447 (1918)

67. Rapaport, D.C.: The Art of Molecular Dynamics Simulation. Cambridge University Press, Cambridge (1995)

68. Richefeu, V., Radjai, F., Youssoufi, M.S.E.: Stress transmission in wet granular materials. Eur. Phys. J. Eng. 21(4), 359-369 (2006)

69. Röck, M., Morgeneyer, M., Schwedes, J., Brendel, L., Wolf, D.E., Kadau, D.: Visualization of shear motions of cohesive powders in the true biaxial shear tester. Partic. Sci. Technol. 26, 43-54 (2008)

70. Roux, S.: Quasi-static contacts. In: Herrmann, H.J., Hovi, J.P., Luding, S. (eds) Physics of dry granular media-NATO ASI Series E 350, Kluwer, Dordrecht, p. 267 (1998)

71. Sadd, M.H., Tai, Q.M., Shukla, A.: Contact law effects on wave propagation in particulate materials using distinct element modeling. Int. J. Non-Lin. Mech. 28(2), 251 (1993)

72. Savkoor, A.R., Briggs, G.A.D.: The effect of tangential force on the contact of elastic solids in adhesion. Proc. R. Soc. Lond. A 356, 103 (1977)

73. Schäfer, J., Dippel, S., Wolf, D.E.: Force schemes in simulations of granular materials. J. Phys. I France 6, 5-20 (1996)

74. Severens, I.E.M., de Ven, A.A.F.V., Wolf, D.E., Mattheij, R.M.M.: Discrete element method simulations of toner behavior in the development nip of the oce direct imaging print process. Granular Matter 8(3-4), 137-150 (2006)

75. Sinkovits, R.S., Sen, S.: Nonlinear dynamics in granular columns. Phys. Rev. Lett. 74(14), 2686 (1995)

76. Spahn, F., Hertzsch, J.M., Brilliantov, N.V.: The role of particle collisions for the dynamics in planetary rings. Chaos Solitons Fractals 5, 1945 (1995)
77. Sperl, M.: Experiments on corn pressure in silo cells. Translation and comment of Janssen's paper from 1895. Granular Matter 8(2), 59-65 (2006)

78. Suiker, A.S.J., Fleck, N.A.: Frictional collapse of granular assemblies. J. Appl. Mech. 71, 350-358 (2004)

79. Tanakov, M.Y., Trusov, L.I., Belyi, M.V., Bulgakov, V.E., Gryaznov, V.G.: Elastically stressed state in small particles under conditions of Hertzian contacts. J. Phys. D 26, 997 (1993)

80. Thornton, C.: Force transmission in granular media. KONA Powder Particle 15, 81-90 (1997)

81. Thornton, C.: Numerical simulations of deviatoric shear deformation of granular media. Géotechnique 50(1), 43-53 (2000)

82. Thornton, C., Antony, S.J.: Quasi-static deformation of a soft particle system. Powder Technol. 109(1-3), 179-191 (2000)

83. Thornton, C., Randall, C.W.: Applications of theoretical contact mechanics to solid particle system simulation. In: Micromechanics of granular media. Elsevier, Amsterdam (1988)

84. Thornton, C., Yin, K.K.: Impact of elastic spheres with and without adhesion. Powder Technol. 65, 153 (1991)

85. Thornton, C., Zhang, L.: A DEM comparison of different shear testing devices. In: Kishino, Y. (ed) Powders and Grains 2001. Balkema, Rotterdam, pp. 183-190 (2001)

86. Tighe, B.P., Sperl, M.: Pressure and motion of dry sand: translation of Hagen's paper from 1852. Granular Matter 9(3/4), 141144 (2007)

87. Tomas, J.: Particle adhesion fundamentals and bulk powder consolidation. KONA 18, 157-169 (2000)

88. Tomas, J.: Fundamentals of cohesive powder consolidation and flow. Granular Matter 6(2/3), 75-86 (2004)

89. Tykhoniuk, R., Tomas, J., Luding, S.: A microstructure-based simulation environment on the basis of an interface enhanced particle model. Granular Matter 8(3/4), 159-174 (2006)

90. Valverde, J.M., Castellanos, A.: Compaction of fine powders: from fluidized agglomerates to primary particles. Granular Matter 9(12), 19-24 (2007)

91. Vermeer, P.A., Diebels, S., Ehlers, W., Herrmann, H.J., Luding, S., Ramm, E. (eds) Continuous and Discontinuous Modelling of Cohesive Frictional Materials. Lecture Notes in Physics, vol. 568. Springer, Berlin (2001)

92. Vermeer, P.A., Ehlers, W., Herrmann, H.J., Ramm, E.: (eds) Modelling of Cohesive-frictional materials, Balkema, Leiden, Netherlands (ISBN 041536023 4) (2004)

93. Walton, K.: The oblique compression of two elastic spheres. J. Mech. Phys. Solids 26, 139 (1978)

94. Walton, O.R.: Force models for particle-dynamics simulations of granular materials. NATO ASI Ser. E Appl. Sci. 287, 367379 (1989)

95. Walton, O.R.: Effects of interparticle friction and particle shape on dynamic angles of repose via particle-dynamics simulation. In: Workshop: Mechanics and Statistical Physics of Particulate Materials (1994)

96. Walton, O.R.: Elastic frictional contact models based on analysis of Mindlin (1949), private communication (1995a)

97. Walton, O.R.: Force models for particle-dynamics simulations of granular materials. In: Guazzelli, E., Oger, L. (eds) Mobile particulate systems. Kluwer, Dordrecht, p. 367 (1995b)

98. Walton, O.R., Braun, R.L.: Viscosity, granular-temperature, and stress calculations for shearing assemblies of inelastic, frictional disks. J. Rheol. 30(5), 949-980 (1986)

99. Zhu, C.Y., Shukla, A., Sadd, M.H.: Prediction of dynamic contact loads in granular assemblies. J. Appl. Mech. 58, 341 (1991) 